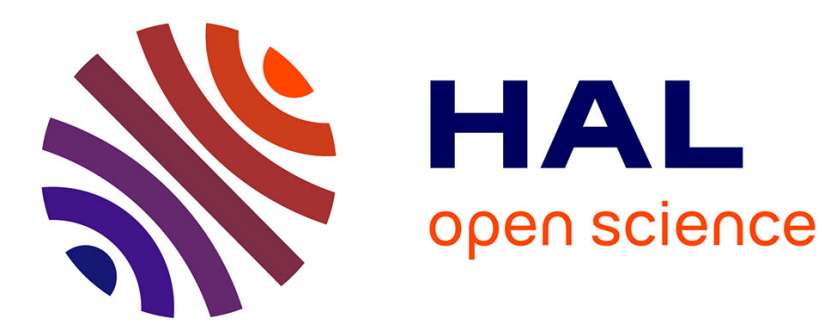

\title{
Virtual Reality: Lessons Learned from WEST Design and Perspectives for Nuclear Environment
}

Lionel Meunier, Delphine R Keller, Pierre Guédon

\section{To cite this version:}

Lionel Meunier, Delphine R Keller, Pierre Guédon. Virtual Reality: Lessons Learned from WEST Design and Perspectives for Nuclear Environment. Fusion Engineering and Design, In press. hal01609929

\section{HAL Id: hal-01609929 \\ https://hal.science/hal-01609929}

Submitted on 4 Oct 2017

HAL is a multi-disciplinary open access archive for the deposit and dissemination of scientific research documents, whether they are published or not. The documents may come from teaching and research institutions in France or abroad, or from public or private research centers.
L'archive ouverte pluridisciplinaire HAL, est destinée au dépôt et à la diffusion de documents scientifiques de niveau recherche, publiés ou non, émanant des établissements d'enseignement et de recherche français ou étrangers, des laboratoires publics ou privés. 


\title{
Virtual Reality: Lessons Learned from WEST Design and Perspectives for Nuclear Environment
}

\author{
Lionel Meunier ${ }^{\mathrm{a}}$, Delphine Keller ${ }^{\mathrm{a}}$, Pierre Guédon ${ }^{\mathrm{a}}$
}

${ }^{a}$ CEA, IRFM, F-13108 St. Paul lez Durance CEDEX, France

From 2012 to 2016, the Tore Supra tokamak was upgraded in an x-point divertor device within the frame of the WEST project. A huge design activity was made to modify the whole configuration of Tore Supra including the invessel components. Some of the most noticeable changes are the addition of inner divertor coils inside the vacuum vessel and the replacement of all plasma facing components.

Fitting all the new components in a previously existing environment was probably one of the major challenges of the project. To improve the assembly plan and the design of toolings, Virtual Reality (VR) was introduced in the design process.

For complex assembly operations, simulations were developed for VR headsets from CAD models. From the requirements expressed by the design team, the VR engineers developed dedicated tools to address the specific issues. Engineers, designers and operators were then able to experience the assembly conditions in a $3 \mathrm{D} 360^{\circ}$ real size immersive environment and interact with the models thanks to a large set of dedicated tools which are developed in the VR lab. This approach showed impressive results for issues such as accessibility, complex kinematics of large components and design of handling tools. It allowed reducing the number of mock-ups and helped the WEST assembly team to reduce the number of unforeseen assembly issues. It was for example used to simulate the assembly of the divertor: insertion through the ports, toroidal displacement on rails, and assembly with gaps lower than $1 \mathrm{~mm}$.

Virtual Reality will also provide innovative solutions to help designers in the anticipation of challenges linked to operation of a nuclear facility: operator's training for nuclear maintenance, virtual mock-ups for qualification of maintenance, maintenance scenario optimization with real time simulations... A first module plotting the dose rate is currently being tested. On the one hand, it would for example allow designers to improve the design by adapting maintenance operations to any complex 3D map of dose rate, and on the other hand, it would permit workers to train for operations in radioactive environments (showing no go areas, hot spots...).

Keywords: WEST, Virtual Reality, Maintenance, Nuclear, Accessibility.

\section{Introduction}

The Tore Supra tokamak has been transformed in an $\mathrm{X}$-point divertor fusion device within the frame of the WEST (W-for tungsten-Environment in Steady-state Tokamak) project, launched in support to the ITER tungsten divertor strategy [1]. The WEST project consists in a major upgrade of the superconducting medium size tokamak Tore Supra with focus to minimize risks for ITER divertor procurement and operation. This modification consists in changing the present circular magnetic configuration to a divertor configuration (see Fig. 1.) and implementing an ITER like actively cooled $\mathrm{W}$ divertor.

The D-shaped plasma of WEST being so different from the original round plasma of Tore Supra (see Fig. 1.), all components facing the plasma were removed: PFC, antennas, bumpers, diagnostic plugs and vacuum vessel actively cooled protection panels. From 2012 to 2016, the project concentrated on designing, manufacturing, qualifying and assembling the new parts $[2]$.

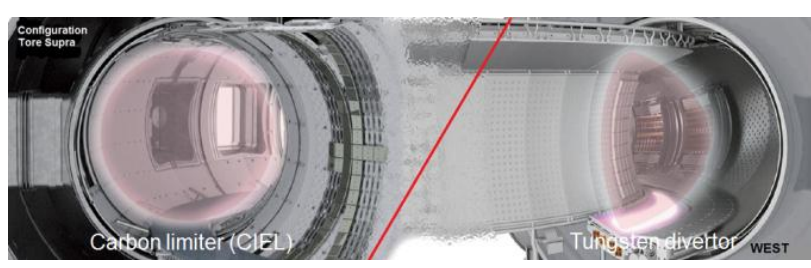

Fig. 1. Tore Supra (left) vs WEST (right) plasma configuration.

Changing so many components, in such a tight and complex environment as a previously existing tokamak was a challenge. During the design phase, it became obvious that assembly would become one of the main concerns for the project. Mounting two $4 \mathrm{~m}$-diameter coils within the vacuum vessel, inserting meters long components through ports designed 25 years ago, assembling divertor sectors with $0.1 \mathrm{~mm}$ gaps were some of the challenges the project team faced.

To face these challenges, virtual reality simulations were developed and a new set of tools was provided to the project to improve the design and plan assembly.

The first part of the paper will describe the major changes in the configuration of WEST compared to Tore Supra. The second part will present the extensive use of virtual reality to perform engineering analysis in support 
to design and assembly and the third part will investigate the potential of this technology with regard to one of the main challenge of fusion: operating nuclear tokamaks.

\section{WEST main components design and assembly}

Tore Supra internal components disassembly was performed in 2013. About 1500 components were removed from the vessel and more than 65 tons of materials were handled. All these components had to be re-designed, manufactured and installed to obtain the new WEST configuration (see Fig. 2.). The installation of the first WEST component took place in October 2014 and end 2016 the first plasma configuration was reached.

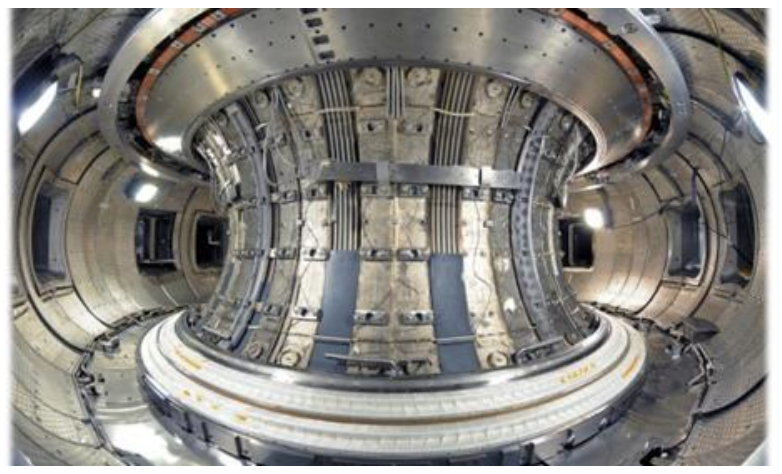

Fig. 2. Inside the vacuum vessel in June 2016. Lower coil winding is still visible.

\subsection{Divertor Coils}

The WEST divertor coils are made up of two stainless steel casings (4 meters diameter - see Fig. 3.) containing a copper winding pack cooled by hot pressurized water (up to $180^{\circ} \mathrm{C}, 4 \mathrm{MPa}$ ). The electrical insulation of the coil conductor is insured by a fiber glass layer impregnated by an epoxy resin. Each casing is welded inside the vacuum vessel [3].

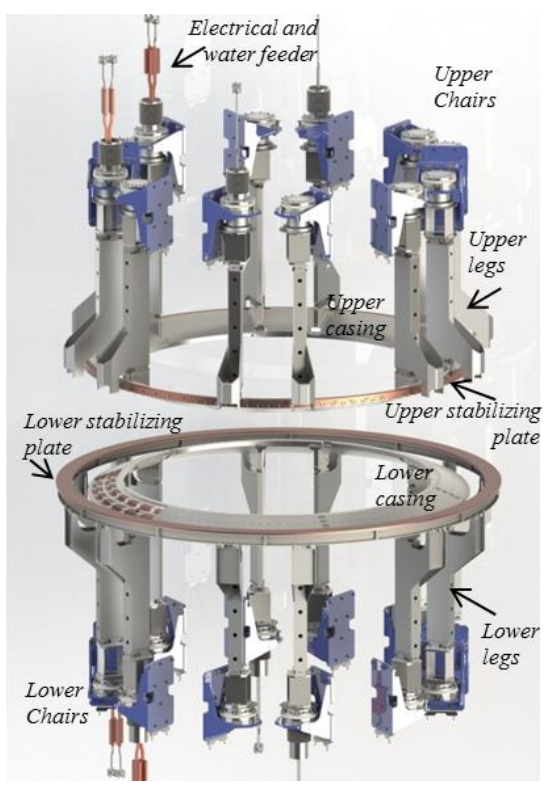

Fig.3. View of the divertor structure and coils assembly

Each $60^{\circ}$ sector of the divertor coils casing was introduced through an equatorial port and bolted to the adjacent sectors before being tightly welded. The two
$8400 \mathrm{~kg}$ rings were then handled and docked to their supporting legs previously fixed on the magnetic iron circuit outside the vacuum vessel.

The upper stabilizing loop sectors had to be brazed together and fixed to the upper casing before lifting it to its final position.

The winding of the pre-formed conductors were then performed. All operations had to be performed inside constrained space of the WEST vacuum vessel. These operations were very challenging as it required the induction brazing (Fig. 4.) and insulation of 140 conductor segments. For each brazed joint, a quality assurance procedure had been set up. Many specific tools had to be developed and 5 months were necessary to successfully complete this complex task. It has to be noted that the two structures were aligned with respect to the magnetic axis of the tokamak within less than one millimeter.

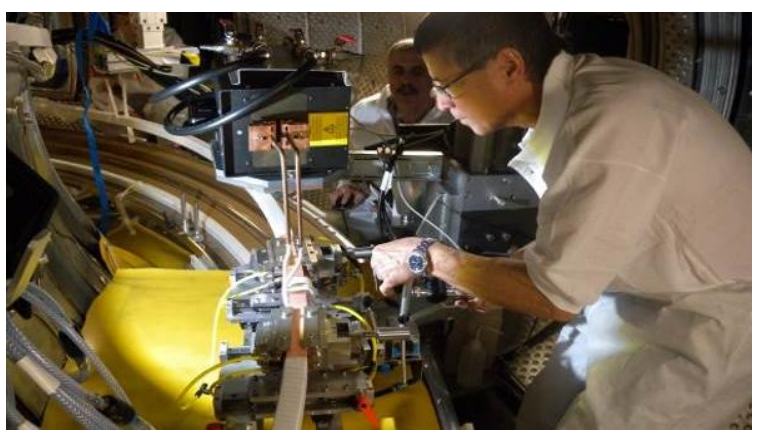

Fig.4. Induction brazing of the divertor coils.

\subsection{Divertor}

WEST nominal divertor element is based on the design of the vertical target of ITER divertor and is representative of the straight high heat flux part of ITER targets (see Fig. 5.). Each WEST element accounts for $35 \mathrm{~W}$ monoblocks $(28 \times 28 \times 12 \mathrm{~mm})$, about $1 / 3$ of ITER element [4].

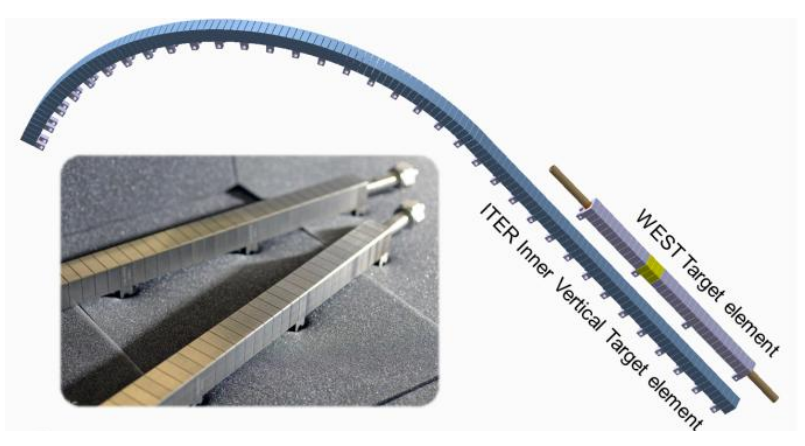

Fig.5. Comparison of ITER vertical target and WEST target elements.

WEST lower divertor target is constituted of 456 elements. In order to start WEST operation and test these prototypes the divertor target will be complemented with non-actively cooled W-coated graphite elements, due to the duration of ITER like component procurement.

\subsection{W-coated Plasma Facing Components}

Due to the time required for the procurement of ITER-like PFC to fully cover the lower divertor target, 
WEST will start with a mix of ITER-like actively cooled elements and inertial elements made of W-coated graphite.

The upper divertor target, symmetric to the lower divertor target, as well as the pumping baffle of the lower divertor and the VDE protections of the upper part of the vessel require new actively cooled PFC. 480 upper divertor elements, 150 baffle PFCs and $26 \mathrm{VDE}$ protections have been manufactured, leak tested and $\mathrm{W}$ coated. Divertor elements and baffle PFCs have been assembled in $30^{\circ}$ sectors (see Fig. 6).

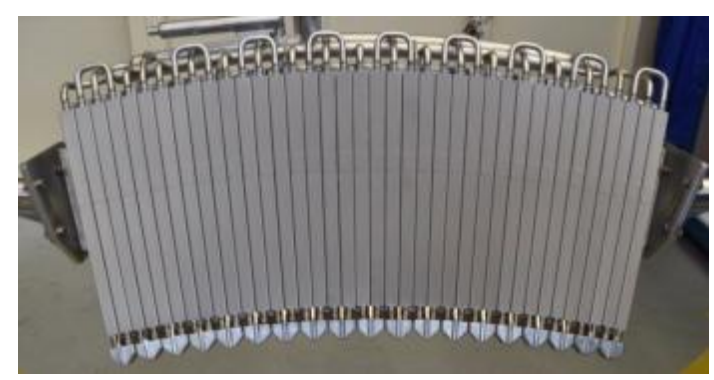

Fig. 6. $30^{\circ}$ upper divertor sector

\section{Virtual reality for WEST}

The WEST project had to face several challenges during assembly:

- existing environment, with its known nominal position and unknown actual position,

- constrained space to introduce components: the ports of Tore Supra have not been changed during the WEST project,

- constrained space to perform many assembly operations such as welding and brazing,

- $\quad$ low tolerances (around $1 \mathrm{~mm}$ ).

Tight scheduleand cost constraints did not allow for much prototyping and assembly trials.

In CEA/Institut de recherche sur la fusion magnétique (CEA-IRFM), Virtual Reality (VR) and 3D simulations were introduced in the design process to bring solutions to those issues [5] [6]. For each study to be performed the best VR tool (among those available in CEA-IRFM VR lab) is selected. Then, dedicated simulations are prepared in close collaboration with the project leader, the designers and the maintenance operators. Most of the time, specific developments are also required in order to accurately answer the problem.

\subsection{Virtual mannequin and motion capture}

The objective is to validate industrial scenario including the operator. The development of advanced simulation technologies and tools to simulate the operator interactions with the environment enable to validate the assembly or maintenance scenario feasibility [7]. To do so, VR peripherals such as motion capture system coupled to a virtual manikin were used. It is coupled with XDE Physics, the interactive physics engine developed by CEA-LIST, featuring accurate collision detection and multibody dynamics interactions. While an operator is performing the simulations, collisions are displayed on the $4 \mathrm{~m} \times 2.5 \mathrm{~m}$ screen, the purpose being to display operator interactions with the pseudo-full size environment.

During the WEST project, it was extensively used to perform accessibility and ergonomics studies for hands on operations with unusual positions (see Fig . 7.).

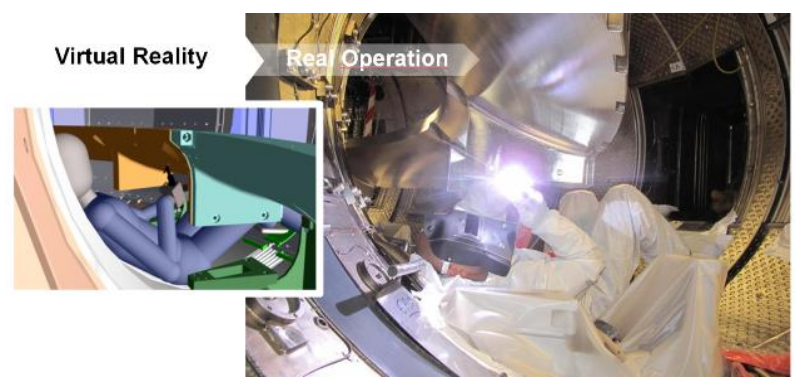

Fig.7. Virtual and real situation of the welding operation on the divertor coils structures of WEST.

This type of simulation shows extremely accurate results: all operations tested in the VR lab with such technique went smoothly during the actual assembly of WEST. However a full body tracking suit is rather long to put on and depending on the situation to study, full body motion tracking is not essential. Instead simpler tools can be used such as those presented below.

\subsection{D immersive headset}

3D immersive headset became very popular in the last years. Several brands offer such device mostly for gaming. For accessibility and maintenance studies, 3D headsets allowed making great improvements, by diplaying $360^{\circ}$ environment, real size, with $3 \mathrm{D}$, and a 12 $\mathrm{m}^{2}$ area where we can walk freely. The accurate tracking of head movement let the user choose naturally its viewpoint. This helps creating a feeling of presence in the scene which is one of the key of this technology: after experiencing the VR scene, the user will remember it almost as if it had been real. The other important consequence is that the user, despite not seeing his body, actually knows its position, as anyone knows the position of his shoulder or elbow without having to look at it. $\mathrm{He}$ is therefore able to judge if the space is appropriate to perform specific operations, if he can reach components, etc...

For WEST project, this type of simulation was performed with CAD models (CREO) imported in Unity3D. To improve the feeling of reality, it is necessary to make a detailed work on textures selections and setup of lights and shades. The models must also be light enough to allow a 90 fps refreshment for the headset (see Fig. 8.). Since 2015, CEA-IRFM worked a lot to automatize this process. The progresses now allow creating a rather complex scene in around 4 hours, where it needed days before.

Once the simulation is ready, the project team members are immersed in the environment and can decide on the technical solutions. As for the virtual manikin simulations described in $\$ 3.1$ interactions are displayed on the screen to allow interactions between the person in the virtual world and the rest of the team. 


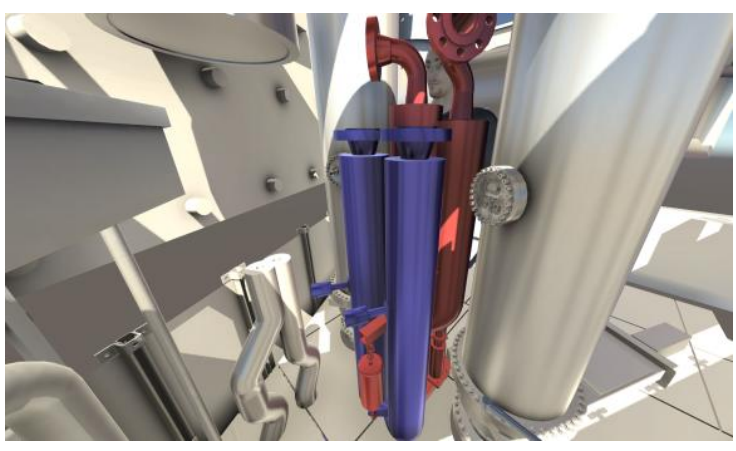

Fig.8. WEST simulation for improvement of flange position to allow their assembly

This kind of simulations showed very good results. It allowed people from different backgrounds to quickly understand the issues and converge on the best practical solution for all. For very complex designs, thanks to the reduction of time needed to prepare a new scene, iterative loops of improvements of the design and update of the virtual simulations were possible. In that case, designers could fully benefit of the advantage of having seen a detailed real scale VR version of the components being designed, in their assembled environment. This brings information comparable to those a real mock-up would. The advantage being that it is available in a matter of hours and almost for free.

\subsection{Interaction with virtual models}

The latest released of 3D headsets include tracked controllers simply held by the operator hands. Those can be used to improve the simulations by adding interactions with the virtual environment. Compared to the full body tracking suit, this latter device is available instantaneously. The controllers also give an access to control specific functions and in particular it can serve as a virtual toolbox to the operator. A wide range of tools was developed by CEA-IRFM VR lab (fig. 9): moving or removing parts, doing cuts, including full size operators and controlling their position, designing simple geometries, getting properties from the CAD models, displacements on large distances... These tools are now included as a package in all new simulation under the 3D immersive headsets.

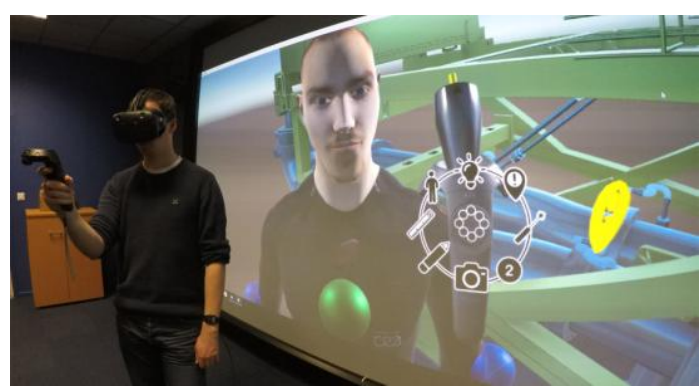

Fig.9. Interaction tools with the virtual model thanks to tracked controllers

Other hardware's are also used for simulation of handling tools. Since those tools usually have limited degrees of freedom, they are easier to control with simpler devices. To check insertion of components in the ports of WEST, simulations with limited degrees of freedom were performed to check for clashes. Once again, the operator was assessing the operation with the 3D immersive headset, which allowed a very quick and efficient check for clashes all around the components. This type of simulation was performed several times to check the design of tooling for the divertor assembly (see Fig. 10. And 11.). It allowed iterative improvements of the tools, and the actual assembly went without any issues, despite the assembly gaps being sometimes around $0.1 \mathrm{~mm}$.

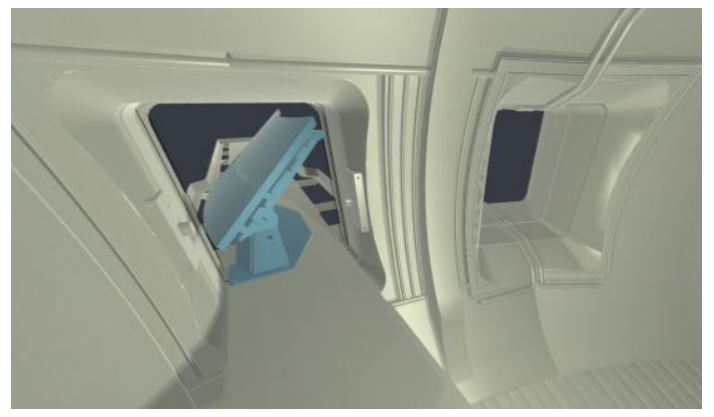

Fig.10. WEST simulation for port insertion

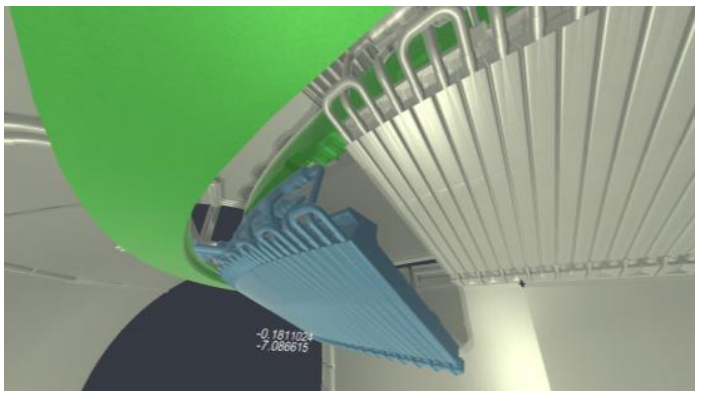

Fig.11. WEST simulation for assembly tooling

For this particular application, as the gaps during port insertion where very small, a cloud of points coming from actual measured position of the port where included in the scene.

\section{Virtual reality for nuclear tokamak}

The work described in the previous sections shows the potential of applications in a nuclear facility:

- on assembly preparation: the impact of assembly on design can be assessed very accurately without physical mock-ups. Development of VR mock-ups would permit to test foreseen assembly scheme very early on with a cost negligible compared to actual mock-ups. It must also be mentioned that VR mockups can cover a much larger scale than physical mockup. One could develop a simulation of a whole rooms or buildings to test assembly scenarios.

- on maintenance: VR can improve both hands-on maintenance (as shown in the previous sections) and remote handling $(\mathrm{RH})$. Devices specific to $\mathrm{RH}$ such as haptic arms are already used in the industry. They allow a more refined interaction with the simulation since it gives force feedback. For hands-on maintenance, CEA-IRFM also developed a vibrotactile suit with ten actuators along the arm [7]. It enhances the perception of collisions with virtual elements is a very promising approach to the promotion of spatial awareness of the operator in a cluttered environment. 
This could prove very useful in a nuclear environment when physical contact sometimes needs to be avoided (not to damage suits, gloves...).

Just as it was the case for WEST, the foreseen maintenance operations could be tested by real operators and their feedback would allow optimizing the design.

- training of operator would also be an obvious application; and given the demonstrations required before an operator can enter a hot area, the benefits could be enormous.

But since anything can be displayed in the simulations, the VR technologies also allow going further. For example physical data such as temperatures or contaminated surfaces could be displayed. In CEAIRFM, tools were developed to be able to display a representation of the dose an operator would receive. The dose was computed with Tripoli software and the values were displayed as a red "fog", more or less thick depending on the dose rate in that area (see Fig. 12.). A dosimeter was also developed which allows computing both the current dose received by the operator given its position in the virtual environment; and the cumulated dose since the beginning of the operations.

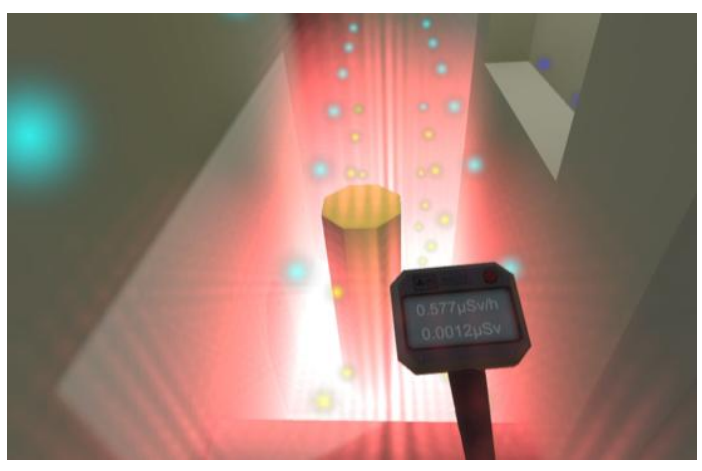

Fig.12. Dose and dosimeter RV simulation

This kind of application of VR could prove very useful as it is always difficult to present dose results which are by nature a 3D data. Usually, they are presented as maps on specific cuts; hence a lot of details are lost. With VR, in addition to being able to show designers and operators the actual dose in all the space, people can experience the environment which is known as one of the best ways to remember it efficiently.

\section{Conclusions and perspectives}

With the fast developments in VR technologies in the last years, CEA-IRFM has been able to include it in its design cycle. Preparing a VR simulation where the team can experience the design "feel" the environment they are working on is feasible in a matter of hours. A package of tools to interact with the virtual world has also been prepared and can be included in any Unity3D environment. However, specific developments are still required for many applications, since no software on the market can efficiently convert complex CAD models to VR scene. Furthermore, those softwares only contain a limited set of functions and do not allow including home-made tools even if sometimes they require just a few hours to be developed. Having a team dedicated to VR developments is therefore still mandatory.

Having already made good progress in the field of VR for accessibility and maintenance in a non-nuclear tokamak like WEST, CEA-IRFM is now starting specific developments for nuclear installations. In the case of ITER maintenance shall be demonstrated before entry into service. This forces the teams to plan for maintenance at early stages of the design when not even the building exists. In that case, VR is bringing a great added value, allowing engineers to 'be' in the conditions where maintenance will be performed. It has already been proved that VR can add information by plotting physical data such as dose rate.

In the future, interactions with the VR simulations will be more and more refined. The hardware currently available only allows very little physical interactions and this showed to be the main limitation when trying to study specific assembly operations for WEST such as welding: the VR lab started developing a VR weld process. But it quickly appeared obvious that more haptic interactions were required since precision welds require welders to support their arm, elbow or whist. The same happens when the environment forces the operator to take an unnatural position using components as supports. The same kind of problem appears when the operator is lifting components: weight, weight repartition, grabbing point... all have an influence on the movements of the operator.

Other aspects that may have a large influence are those linked to the emotional state of operator: how stress to create stress in VR, how does it influence the operators and the conclusions of the studies. It may prove crucial in nuclear facilities.

\section{References}

[1] A. Grosman et al., The WEST programme: Minimizing technology and operational risks of a full actively cooled tungsten divertor on ITER, Fusion Engineering and Design 88 (2013) 497-500.

[2] J. Buccalossi et al., Progresses on WEST Platform Construction towards First Plasmas, 26th IAEA Fusion Energy Conference (2016).

[3] L. Doceul et al., Qualification, manufacturing and assembly of the WEST divertor structure and coils, Fusion Engineering and Design (2016).

[4] M. Missirlian et al., The WEST project: Current status of the ITER-like tungsten divertor, Fusion Engineering and Design 89 (2014) 1048-1053.

[5] D. Keller et al., Use of virtual reality for optimizing the life cycle of a fusion component, Fusion Engineering and Design (2014).

[6] A. Pilia et al., Application of virtual reality tools for assembly of WEST components: Comparison between simulations and physical mockups, Fusion Engineering and Design, 98-99 (2015), 1589-1592.

[7] C. Louison et al., Operators' accessibility studies for assembly and maintenance scenarios using virtual reality, Fusion Engineering and Design (2016). 